\title{
L’utilisation Des Structures De Soins Dans La Région Du Logone Occidental Au Sud Du Tchad, Un Défi Pour La Gouvernance En Santé
}

\author{
Djimouko Sabine \\ Département de Géographie, Université de Moundou, Tchad \\ Dr Madjigoto Robert \\ Département de Géographie, Université de N’Djaména, Tchad \\ Nguendo-Yongsi H.B. \\ Institut de Formation et de Recherche Démographiques (IFORD), \\ Université de Yaoundé II, Cameroun \\ Tchotsoua Michel \\ Professeur titulaire des Universités, Département de Géographie, \\ Université de N’Gaoundéré, Cameroun
}

doi: 10.19044/esj.2016.v12n30p89 URL:http://dx.doi.org/10.19044/esj.2016.v12n30p89

\begin{abstract}
Background: Health situation in Chad, particularly in the Western Logone is characterized by a general high morbidity, a difficult access to healthcare structures, and by a plurality of healthcare provision. In the evnt of illnesses, very few patients (18\%) resort to modern healthcare. Objective: This study aims at adressing the link between the provision of healthcare system, geographical accessibility and patients therapeutic behaviors . Methods: We have resorted to methodology usually used in in geography that is review literature, surveys and interviews. Over the 429 investigated households, a response rate of $99.9 \%$ was obtained.. Levels of analyses include both descriptive and explanatory analyses. Results: $96 \%$ of the investigated individuals live close to a health center $(0$ to $10 \mathrm{kms})$ is. Conversely, 75\% are attending healthcare centers located beyond $10 \mathrm{kms}$. In the event of illnesses, about $82 \%$ of patients resort firstly to traditional care.
\end{abstract}

Keywords: Provision of health, healthcare structures, accessibility, health governance, Chad

Résumé

Contexte : le contexte sanitaire du Tchad, et plus particulièrement celui de la région du Logone occidental, est caractérisé par une forte 
morbidité, des conditions d'accès complexes et une pluralité de l'offre de soins. En cas de maladies, peu de patients (18\%) se présentent dans un centre de santé moderne. Objectif : cet article recherche le lien qui existe entre l'organisation des soins, l'accessibilité et les comportements des patients. Méthode et matériels : afin de valider nos hypothèses, nous mobilisons la méthodologie classique en géographie associant recherche documentaire, travail cartographique, enquêtes et entretiens. L'enquête touche 429 ménages, le taux de réponse est de 99,9\%. Les données ont été saisies sous le Logiciel SPSS version 20. Elles ont fait l'objet d'une analyse descriptive et explicative. Résultats : la proportion de la population qui habite à proximité des centres de santé, c'est-à-dire, dans un rayon de 0 à $10 \mathrm{~km}$ est de $96 \%$. Inversement, $75 \%$ des patients fréquentent les centres de santé situés au-delà de $10 \mathrm{~km}$ laissant après eux des centres de santé les plus proches vides. Egalement, 82\% s’adonnent aux traitements traditionnels après un épisode de maladie.

Mots-clés. Organisation des soins, centres de santé, accessibilité, gouvernance de santé, Tchad

\section{Introduction/problématique}

Depuis plus de trois décennies, la question de la santé et des moyens de la préserver ont fait l’objet de plusieurs débats. En Afrique subsaharienne, le secteur de la santé a été tenu, pendant longtemps, à l'écart des programmes de développement. Seuls les secteurs agricole et industriel étaient privilégiés (Djimouko, 2008). Conscients du fait que le lien entre la santé et le développement n’a pas été bien établi au début des indépendances, les États africains ont opté pour la réorientation de la politique de développement de l'Afrique en général, et de celle du secteur de la santé en particulier. Leur adhésion massive aux principes des Conférences d'Alma Ata en 1978 et près d'une décennie plus tard, à l'Initiative de Bamako en 1987 témoigne de leur bonne volonté.

Suite à ces engagements pris, des efforts considérables axés sur la construction des infrastructures et la formation du personnel avaient été fournis, en vue d'améliorer la qualité de la santé des populations. Cependant, on constate en général qu'au Tchad et en particulier dans la région du Logone occidental, les indicateurs de santé de la population sont moins reluisants, alors que les centres de santé mis en place à grand frais ne sont pas fréquentés. La grande partie des populations (68\%) n’a pas accès à des soins de santé de base (INSEED, 2014). En effet, les centres de santé n’attirent pas les consommateurs, le système de santé présente sans doute des faiblesses. Les raisons avancées par les consommateurs des services de santé pour justifier la sous-utilisation des centres de santé sont notamment: 
l'éloignement géographique, le manque de moyens financiers, la médiocrité de soins, l'insuffisance du personnel qualifié ou le manque de disponibilité vis-à-vis des patients, mais surtout la proximité des agents du secteur informel de la santé et ceux de médecine traditionnelle. Par conséquent, dans la région du Logone occidental, chaque année, les rapports relèvent une forte morbidité et un faible accès aux soins : les taux d'utilisation des services de soins modernes restent très faibles: environ $11 \%$ en 2010 pour les accouchements, $17 \%$ en 2012 et $20 \%$ en 2013 pour les consultations curatives générales (DRSLOC, 2013).

Selon Djimouko (2008), la sous-utilisation des centres de santé s'explique par le fait que le système de santé de la région d'étude ${ }^{1}$ a, pendant longtemps, été tenu à l'écart de toutes les évaluations, et a ainsi évolué dans l'anarchie, sans faire l'objet d'une étude scientifique, en l'absence de toute vision prospective. Cette situation a largement favorisé la mauvaise répartition des ressources de santé et une faible réponse à la demande des patients (Brunet et Jailly, 2003) : les centres de santé n’offre qu'une réponse partielle aux besoins de la population parce que les services proposés aux patients ne répondent pas à leurs aspirations. En réponse, ces derniers développent un désintéressement vis-à-vis de l'offre et adoptent leurs propres stratégies de recours aux soins. Face à une politique sanitaire qui s'évertue depuis plus de deux décennies à rechercher les outils efficaces pour une meilleure utilisation des centres de santé, et une population qui malgré les efforts fournis afin de favoriser l'amélioration de la qualité de la santé développe une réticence quant à la consommation de l'offre, il faut noter un dysfonctionnement. Le constat de ce dysfonctionnement suscite la question suivante : comment est organisé le système des soins dans la région du Logone occidental et comment les centres de santé sont utilisés par la population? Deux questions subsidiaires structurent la première comme suit : l'organisation de l'offre de soins basée sur la multiplication des centres de santé et le rapproche des soins de la population dans la région du Logone occidental permet-elle à ces derniers de tirer profit ? Le rapprochement des centres de santé offre-t-il aux patients la facilité dans la fréquentation des services de santé moderne?

Dumont (2008), pour sa part, pense que la géographie est le meilleur instrument de mesure qui aide à la compréhension des faits spatiaux. Selon Vigneron (2000), l'approche géographique de la santé conduit le géographe à mesurer d'abord les besoins, puis à apprécier le niveau de l'offre. Cet article s'inscrit dans les études en géographie des soins. Il se veut un outil scientifique d'évaluation du système de santé accordant une place de choix à

\footnotetext{
${ }^{1}$ Selon Dondo en1994, le système de santé de la région du Logone occidental est calqué sur le système de santé national.
} 
l'approche géographique. À notre connaissance, des réflexions ont été menées sur la question de la perception et la déperdition des patients au Tchad, mais pas sur la région d'étude et moins encore sur l'offre de soins. Ces études portent un caractère évaluatif et non scientifique.

Cet article se présente comme un outil d'évaluation du système de soin de la région du Logone occidental : il analyse les différentes raisons qui ont motivé l'utilisation des centres de santé et fait ressortir ensuite dans une analyse descriptive et explicative les variables essentielles qui peuvent motiver le choix d'un type de soin et d'un centre de santé. Le poids de chaque variable dans le phénomène étudié est considéré comme facteur explicatif de l'utilisation des services de santé, ce qui permet de mieux réajuster l'offre face aux besoins réels des consommateurs en vue d'une meilleure utilisation des services de santé.

\section{Cadre méthodologique Cadre conceptuel}

La question d'utilisation des services de santé a fait l'objet d'un nombre important d'études et de recherches. Cette notion d'utilisation est perçue différemment selon les auteurs et selon les milieux, il est considéré comme un concept. Selon Richard (2001), l'utilisation d'un service de santé est conditionnée par son accessibilité. Ainsi, l'utilisation des centres de santé, leur fréquentation et le recours aux soins sont des notions indissociables (Djimouko, 2008). Son analyse entraîne à relever la liste des lacunes du système de santé. La médiocrité de la desserte médicale, la longueur de la distance à parcourir par les patients, le manque de moyens de transport et de communication, l'absence de l'assurance maladie pour les démunis, la cherté des coûts de soins, les longues files d'attente, l'absence de la qualité des soins mais aussi les pesanteurs socioculturelles, la proximité, la disponibilité des tradi-praticiens et autres praticiens de la médecine informelle influençant la décision du patient. Selon Pichéral (1984), la santé est composée de trois dimensions : les besoins, la disponibilité de l'offre et sa consommation, et le recours aux soins, ce qui renvoie à l'utilisation des services de santé et les conditions d'accès aux soins.

Dans le cadre de cette étude, l'utilisation des centres de santé est analysée comme une dimension de la santé dans le contexte de l'existence des obstacles, de barrières des opportunités qui rendent faciles ou difficiles l'aboutissement au lieu de service en vue de son utilisation. L'utilisation des services de soins est effective quand le patient s'approprie le service et pour en faire usage. Cette appropriation témoigne l'accès aux soins. Dans ces travaux, l'accès aux soins de santé, l'utilisation des services de santé et la fréquentation des centres de santé sont utilisés pour exprimer le contact avec l'offre de soins ou l'accès aux soins. Notre travail s'intéresse aux conditions 
pouvant prédisposer un patient à la consommation des services de santé.

\section{Cadre de l'étude : la région du Logone occidental, un milieu difficile de par son caractère géographique}

La région du Logone occidental présente beaucoup de caractéristiques communes avec ses voisines qui faisaient partie d'un espace plus vaste appelé le « Moyen Logone » (Cabot, 1965), limité au Nord entre les parallèles $8,5^{\circ}$ et $11^{\circ}$ et les méridiens $14^{\circ}$ et $17^{\circ}$ Est. L'étendue du Moyen Logone englobait les limites des anciens royaumes du Baguirmi, du Bornou et les Lamidats Foulbé de l'Adamaoua. Le Bas-Logone regroupait les groupes MassaYaéré, l'empire du Bornou et les tribus Mousgoum ou Kotoko. Entre le Logone et les affluents de la Bénoué, on trouve le Lamidat de Reî Bouba et le groupe Gambaye-Laka. Cet espace a été connu grâce aux travaux de Cabot J. en $1965^{2}$.

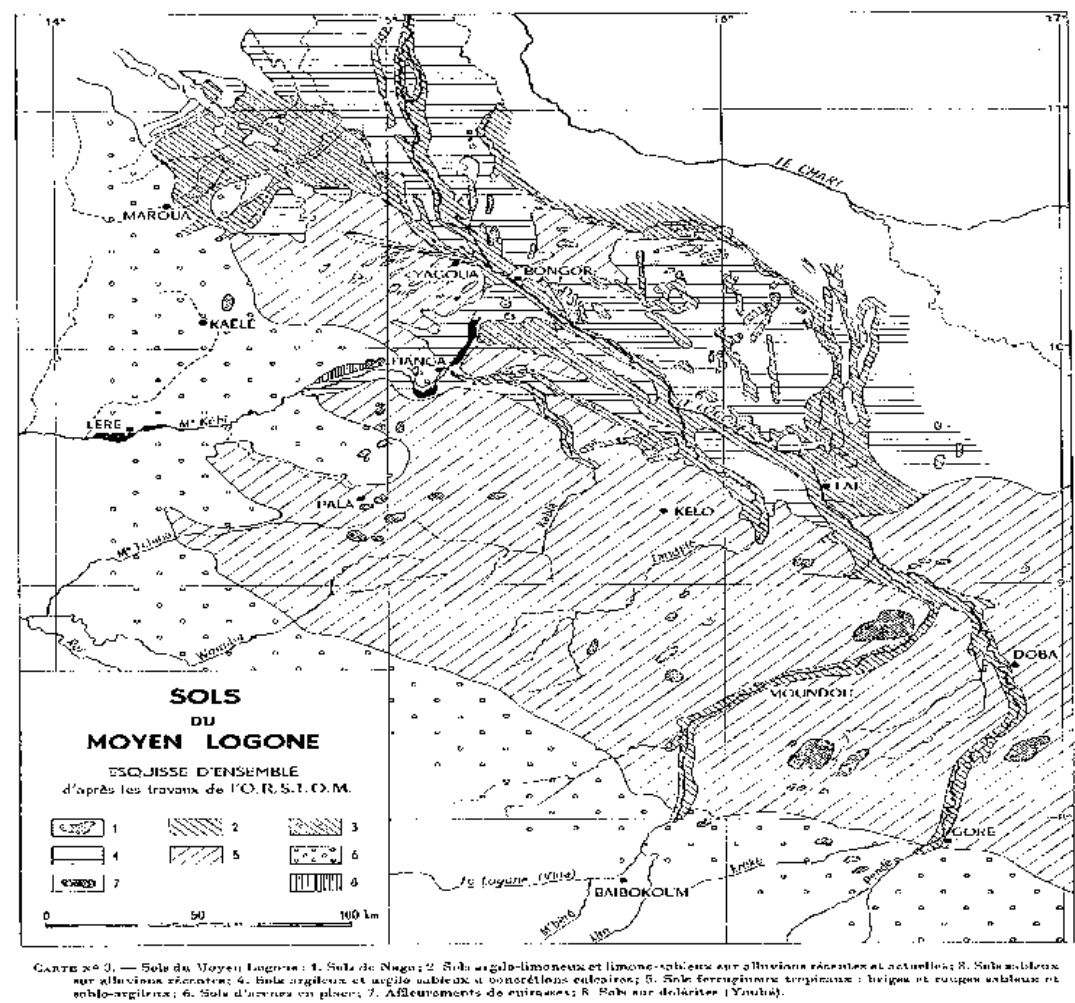

Source : Cabot Jean, 1965

Figure 1. Région du Moyen Logone

Le Moyen Logone (figure1) regroupait des individus présentant des traits communs avec des affinités communes (Cabot, 1965).De nos jours, les

${ }^{2} \mathrm{La}$ carte ci-dessus est une représentation de la région d'étude, elle est tirée de la thèse de Cabot J. intitulée « le Moyen Logone » soutenue en 1965. 
Massa, Mousgoum, Toupouri, Moundang, Foulbé, et Laka sont devenus camerounais ou tchadiens après les indépendances au gré du colonisateur, selon qu'ils se trouvaient à l'Ouest ou à l'Est de la frontière fixée. La région du Logone après les indépendances est devenue territoire tchadien, son histoire est assimilée à celle du pays Sara qui regroupe les quatre anciennes préfectures du Sud du Tchad à savoir, le Moyen-Chari, les deux Logones et la Tandjilé. Elle a une superficie de $8916 \mathrm{~km}^{2}$, sa population est estimée à 865000 en 2013 (DSRLOC, 2013). Les groupes qui y vivaient sont rassemblés sous le vocable de Sara et ont en commun des dialectes, des modes de vie et des coutumes. Ces groupes se rassemblent autour des chefs religieux. L'économie du pays Sara était basée sur une vie de seminomadisme qui permettait de pratiquer l'agriculture et l'élevage. Après l'éclatement du pays Sara, le Logone occidental est administrativement divisé en quatre départements (figure 2):

Le département de Lac Wey (chef-lieu Moundou);

Le département de la Dodjé (chef-lieu Baînamar) ;

Le département de Ngourkousso (chef-lieu Benoye) ;

Et enfin le département de Gueni River (chef-lieu Krim-Krim).
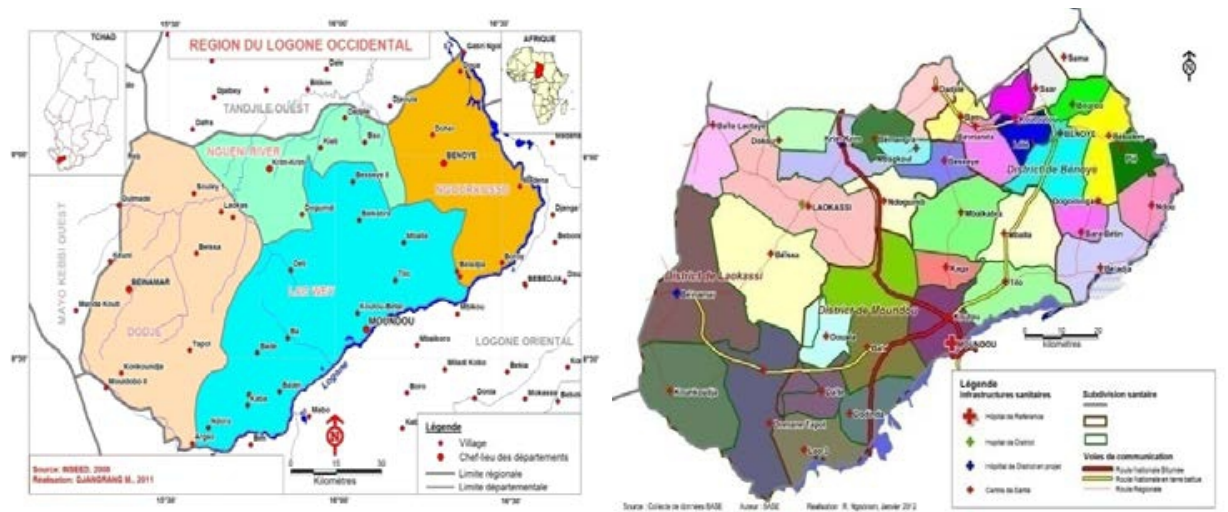

Figure 2. Région du Logone occidental

Figure 3. Organisation des soins en zones de responsabilité

En ce qui concerne l'organisation des soins, la région est découpée en cinq districts sanitaires dont trois fonctionnels ${ }^{3}$. Chaque district sanitaire regroupe en son sein les zones de responsabilités ${ }^{4}$ (Figure 3).

- $\quad$ District sanitaire de Moundou

- $\quad$ District sanitaire de Benoye

- $\quad$ District sanitaire de Laoukassy

${ }^{3}$ La région du Logone occidental doit bénéficier de cinq district sanitaires (district sanitaire de Moundou, de Benoye, de Laoukassy sont opérationnels tandis que ceux de Krim-Krim et de Baînamar sont en gestation).

${ }^{4}$ Les zones de responsabilités sont des découpages des territoires sanitaires qu’on observe sur la figure. 
- $\quad$ District sanitaire de Krim-Krim ;

- $\quad$ District sanitaire de Baïnamar.

La région du Logone occidental dans son ensemble est un milieu qualifié de difficile par Cabot (1965) de par son caractère géographique: son climat chaud ${ }^{5}$, tantôt sec et tantôt humide et la nature inondable de ses sols favorise la propagation et prolifération des germes pathogènes, vecteurs des maladies microbiennes et bactériennes dont souffre la majorité de ses habitants. La région est dotée d'une végétation dominées par de grandes herbes, habitat des insectes nuisibles dont particulièrement les moustiques et mouches tsétsé respectivement vecteurs du paludisme et de la trypanosomiase. Une morphologie difficile, des sols argileux, cassants et gonflants à cause des nodules de calcaire qu'ils contiennent; de nombreuses flaques d'eau pendant la saison des pluies. Un tel cadre peut compromettre le choix de se mouvoir sur une plus ou moins grande distance, afin d'accéder à l'offre de soins. Cette difficulté limite l'utilisation de l'offre de soins pour la population issue des villages enclavés et difficile d'accès, surtout, pendant les saisons des pluies.

\section{Données et méthode}

Afin de répondre aux questions de recherche posées, nous mobilisons la méthodologie classique en géographie associant la recherche documentaire, le travail cartographique, le choix des variables et indicateurs permettant d'orienter la recherche, de repérer et de circonscrire plus facilement les faits observables qu'il faudra analyser. Dans cette étude, il est question de vérifier la relation entre l'organisation des soins, le recours et l'accès aux soins de base.

Afin de nous permettre de mesurer l'accès métrique ou la distance d’accès, nous affectons deux modalités à la variable longueur du trajet. : Le chiffre « 1 » représente les centres de santé qui se trouvent entre 0 et $10 \mathrm{~km}$, donc accessible théoriquement. Le chiffre « 2 » représente ceux qui se trouvent au-delà de $10 \mathrm{~km}$ donc non accessible $(\mathbf{Q 1 7})^{\mathbf{6}}$. Ces deux modalités sont la mesure de la distance standard qui doit séparer le patient de son établissement, selon la norme établie par l'État tchadien. Pour ce dernier, tout établissement qui se trouve à $10 \mathrm{~km}$ du patient est accessible. Cette accessibilité métrique que nous propose l'État tchadien ne prend pas en compte les autres facteurs (milieu de résidence du patient, facilité ou

\footnotetext{
${ }^{5}$ La région du Logone occidental jouit d'un climat tropical soudano guinéen à deux saisons : la saison des pluies s'installe à partir du mois de d'avril et prend fin en octobre. La température moyenne est $30^{\circ} \mathrm{C}$. La hauteur des pluies varie entre 1000 à $1200 \mathrm{~mm}$.

${ }^{6}$ Le questionnaire qui a servi à la rédaction de cet article se trouve en annexe $\mathrm{N}^{\circ} 1$ de la thèse en cours de rédaction par Djimouko Sabine sous la direction du Professeur Tchotsoua Michel
} 
difficulté que rencontrent les patients lors de la recherche de soins). Afin de mieux cerner les enjeux autour de la demande de soins, Notre travail introduit des variables susceptibles d'être des obstacles que les patients rencontrent dans leur déplacement (qualité de soins (Q23), moyen de transport (Q18), statut sociale des patients (Q25-30), image du lieu d'implantation des centres de santé (Q 15), présence des cours d'eau, coupures des routes ou des ponts en saisons des pluies, des routes dégradées (Q19)). D’autres variables comme le temps (Q16) et le coût de déplacement (Q20) permettent d'apprécier le niveau d'accès aux soins.

Au total, 429 ménages tirés sur un total 3344 ont fait l'objet d’échantillonnage. 428 sur 429 ont été enquêtés en juillet 2013 avec succès donnant un taux de réponse de 99,9\%.

Les données ont été saisies sous le logiciel SPSS version 20. Elles ont fait l'objet d'une analyse descriptive et explicative. Pour la spatialisation des données, nous avons utilisé le logiciel Arcgis 10.1. Pour ce faire, nous avons au préalable, transformé nos données de différents formats (MapInfo, Excel, etc.) en Shapes files utilisables sous Arcgis. Nous avons pris le soin de les mettre toutes dans un même système de projection WGS 84.

\section{Résultats et discussions}

\section{Analyse du système de santé de la région : un réajustement du besoin face à l'offre de soins}

Pendant la période coloniale, les principaux problèmes de santé dépistés au sein de la population étaient la lèpre, la trypanosomiase, la bilharziose. Cependant, les autres maladies comme la pneumonie, la méningocoque, la tuberculose, les infections sexuellement transmissibles (IST), le filaire restent en dehors de la prophylaxie des services de santé, tout comme la santé maternelle et infantile laissant le grand fléau de la mortalité maternelle et infantile faire son bonhomme de chemin. De nos jours, les principaux problèmes de santé des populations dans la région du Logone occidental sont par ordre d'importance le paludisme, les infections respiratoires aiguës, la diarrhée, les infections cutanées et les accidents des voies publiques. Les inégalités de santé sont des faits observables dans cette région car il existe des catégories de personnes et de milieux plus vulnérables que d'autres. Pour évaluer l'état de santé de nos enquêtés, nous avons effectué le croisement des variables épidémiologiques. Nous avons eu à demander si au cours des 30 derniers jours qui ont précédé l'enquête, si un membre de la famille au moins a eu à connaitre un épisode de maladie.

L’objectif de l'étude est de pouvoir déterminer les facteurs du risque morbide, mesurer l'ampleur de la morbidité. Sur l'ensemble des ménages enquêtés (429), durant les 30 derniers jours qui ont précédé l’enquête, $87,24 \%$ des familles affirment avoir eu un épisode de maladie dans cet 
intervalle de temps. La proportion des ménages ruraux ayant connu un épisode de maladie est de 84,07\%. Autrement dit, un peu plus de 8 ménages sur 10 ont vu un des leurs souffrir de maladie en milieu rural, alors qu'en milieu urbain, il s’agit de 89,84\% soit 9 ménages sur 10 . Cela veut dire qu'en dehors de la présence de la forte morbidité dont sont victimes les ménages, ceux issus du milieu urbain sont plus vulnérables. Une telle prévalence de la morbidité doit se traduire par la demande de soins, et cela peut être une expression du poids des besoins que les centres de santé doivent satisfaire.

La politique nationale de la santé (PNS) de 2007 à 2015 a pour objectif de favoriser l'accès aux soins de santé pour tous en vue de la réduction sensible de la mortalité et de la morbidité. C’est pour cette raison qu'il y a une multiplication des centres de santé dont le rôle est la prévention des maladies et la promotion de la santé individuelle et collective (Ministère de la Santé Publique, 2015).

Un centre de santé doit assurer la desserte médicale d’environ 5000 à 10000 habitants vivant dans une aire géographique d'un rayon de 0 à $10 \mathrm{Km}$ selon le Ministère de la Santé Publique (2012), soit deux heures de marche. Les zones de responsabilités sont administrées par des districts sanitaires qui ont au-dessus d’eux des délégations régionales de santé. Le district sanitaire est le $2^{\mathrm{e}}$ niveau dans l'échelon du système de soins. Il doit assurer à travers les activités de ses centres de santé une couverture médicale d'au moins 50000 à 150000 habitants. Son aire géographique doit s’étaler au niveau départemental et la direction du district ou de l'hôpital du district doit être implantée dans le chef-lieu du département. Les services de santé comprennent des centres de santé, des hôpitaux, des centres hospitaliers universitaires (CHU) qui peuvent être des structures de soins publiques, privées et traditionnelles.

Afin de mieux gérer les patients, le système de santé moderne est organisé en échelons (hiérarchie).Dans cette hiérarchisation les patients bénéficient d'une organisation préétablie qui les renvoie si besoin se fait sentir, des services de santé de base vers les services de plus en plus spécialisés et qualifiés. Ce niveau d’organisation appelé système de référence permet d'offrir les prestations de base généralement bon marché et proche d'une part, et d'autre part, d'éviter l'encombrement des hôpitaux et des spécialistes avec des cas bénins. Le système de soins, s’il est bien organisé, permettrait de canaliser les malades selon les filières préétablies. De nos jours, les centres de santé sont moins fréquentés que les services des hôpitaux et les services des urgences. Les premiers soins sont administrés par les structures précitées et les soins préventifs ne sont presque pas pratiqués en dehors des programmes ponctuels de vaccination laissant les centres de santé vides. 
La région du Logone occidental est théoriquement la région la mieux dotée en infrastructures de santé après celle de N’Djaména. La distance moyenne qu'un patient doit parcourir pour accéder à un centre de santé dans cette région est de $7 \mathrm{~km}$. D'après le Ministère de la Santé Publique, cette distance est largement en dessous de la norme nationale d'accessibilité géographique qui est de $10 \mathrm{~km}$. Sur 50 zones de responsabilité dont dispose la région, 46 soit 92\% sont opérationnelles en 2013 (DSRLOC, 2013). Par conséquent, la couverture géographique ${ }^{7}$ et la disponibilité physique des établissements de santé sont assurées pour un grand nombre de ménages (96\%). Cependant, quelle est la proportion de la population qui a réellement accès aux soins dans le temps et dans l'espace ? Quels sont les déterminants d'accès. L'accès dépend-il de la présence physique? Ou bien, faut-il considérer d'autres paramètres ?

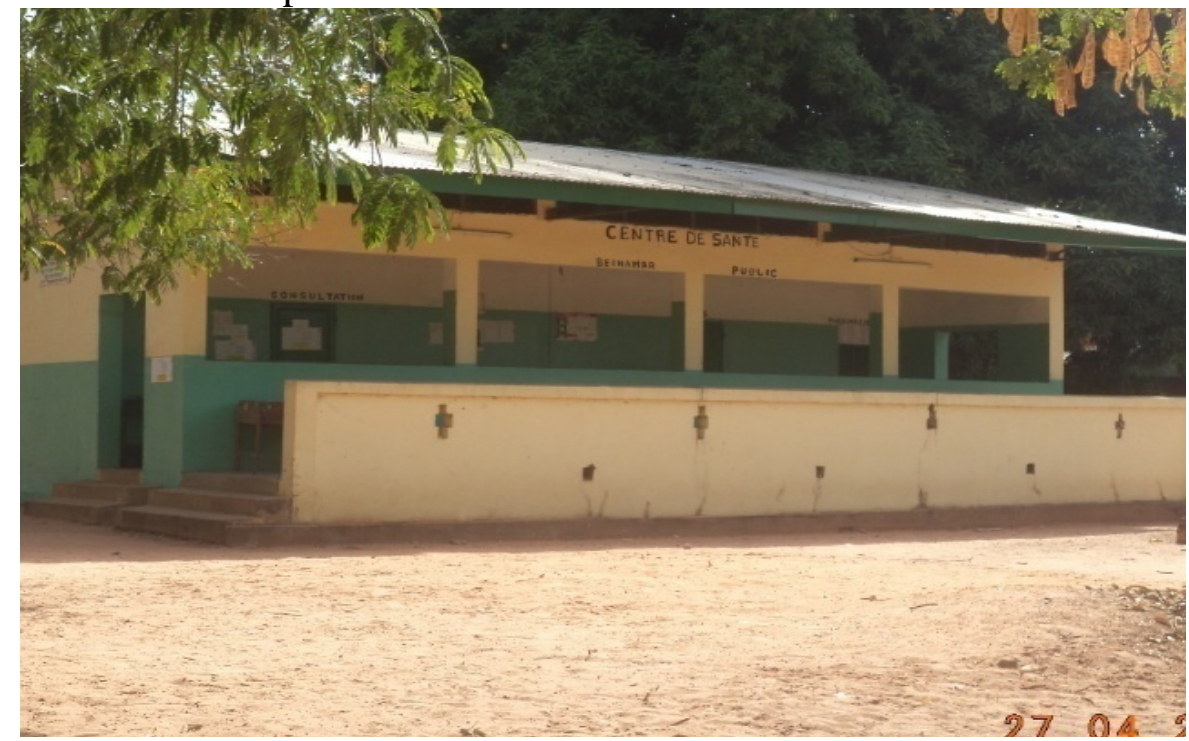

Photo 1. Centre de santé Baïnamar-Ouest (cliché Nadjitoingar D Fabien, Avril 2016).

Le 24 Avril 2016 à 15h 03mn, le dispensaire est déjà désert. Normalement, le service s'arrête à 18h. Ce vide témoigne bien le manque d'intérêt accordé à la fréquentation des centres de santé modernes.

\section{Un recours aux soins dominé par les soins traditionnels}

Selon l'Organisation mondiale de la santé (OMS), 80\% des intentions des patients en Afrique est de voir le tradipraticien (OMS, 2014). Dans la région du Logone occidental, la représentation sociale de la santé joue sur le choix thérapeutique du patient. En effet, Plus de $80 \%$ des habitants

${ }^{7}$ La couverture géographique est le rapport entre le nombre d'établissements sanitaires et par unité administrative d'une part, la population et la superficie de l'unité administrative d'autre part. 
commencent leur premier parcours thérapeutique (soins primaires) par les produits traditionnels (figure4).

Tableau 1 : types de soins et niveaux d'instruction

\begin{tabular}{|c|c|c|}
\hline \multirow[b]{2}{*}{$\begin{array}{c}\text { Niveau } \\
\text { d'instruction }\end{array}$} & \multicolumn{2}{|c|}{ Types de soins } \\
\hline & $\begin{array}{c}\text { Traditionnels } \\
\text { en (\%) }\end{array}$ & $\begin{array}{c}\text { Modernes } \\
\text { en (\%) }\end{array}$ \\
\hline Supérieur & 88 & 12 \\
\hline Secondaire & 82 & 18 \\
\hline Primaire & 81 & 19 \\
\hline Analphabète & 82 & 18 \\
\hline
\end{tabular}

Source : enquête ménage, 2013

u soins traditionnels $\square$ soins modernes

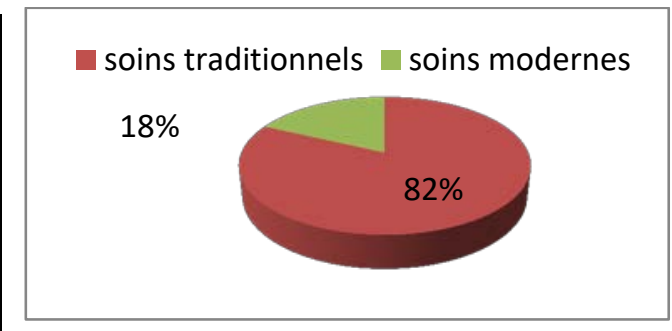

Figure 4. Choix du $1^{\mathrm{er}}$ soin Source : enquête ménage 2013

Généralement, en cas de maladie, les patients utilisent soit des produits traditionnels, soit des produits modernes. Ceux qui utilisent les soins traditionnels comme premier recours sont très nombreux (figure 4). Selon nos enquêtés, la médecine traditionnelle est jugée très efficace et plus proche des patients ; elle est plus sollicitée pour les maladies dites naturelles, (hémorroïde, diarrhée, prostate, paludisme, maladies fongiques et épidermiques).En dehors de ces maladies dites naturelles, les problèmes de santé perçus comme des attaques de la sorcellerie et des divinités sont également traités chez les praticiens des soins traditionnels. Le niveau d'instruction n'est pas une variable qui prédispose au recours à un type de soin moderne. Au regard de nos résultats (tableau1), Les patients analphabètes, ainsi que ceux d'autres niveaux d'instruction adoptent le même comportement en ce qui concerne le choix thérapeutique au $1^{\mathrm{er} e ́ c h e l o n}$ (tableau 1). Le résultat de l'étude contredit celui de Nadjihoroum (2007) qui affirmait que le niveau intellectuel du moins, celui d'une femme peut la prédisposer au recours aux soins de santé prénatale et post natale. Selon le test de khi deux effectué sur nos données, le type de premier soin n'est pas significativement associé au niveau d'instruction dans la région d'étude. On peut également lire que les individus de niveau supérieur sont les plus nombreux à ne pas solliciter les soins modernes au $1^{\mathrm{er}}$ recours (tableau 1 ).

La médecine moderne fait face à la rude concurrence des autres prestataires de soins. Dans l'espace de santé se trouvent les tradi-praticiens, les agents du secteur informel de la médecine moderne, les voyants, marabouts ou exorcistes et accoucheuses traditionnelles. La figure 5 cidessous présente différentes offres de soins présents dans la ville de Moundou. 


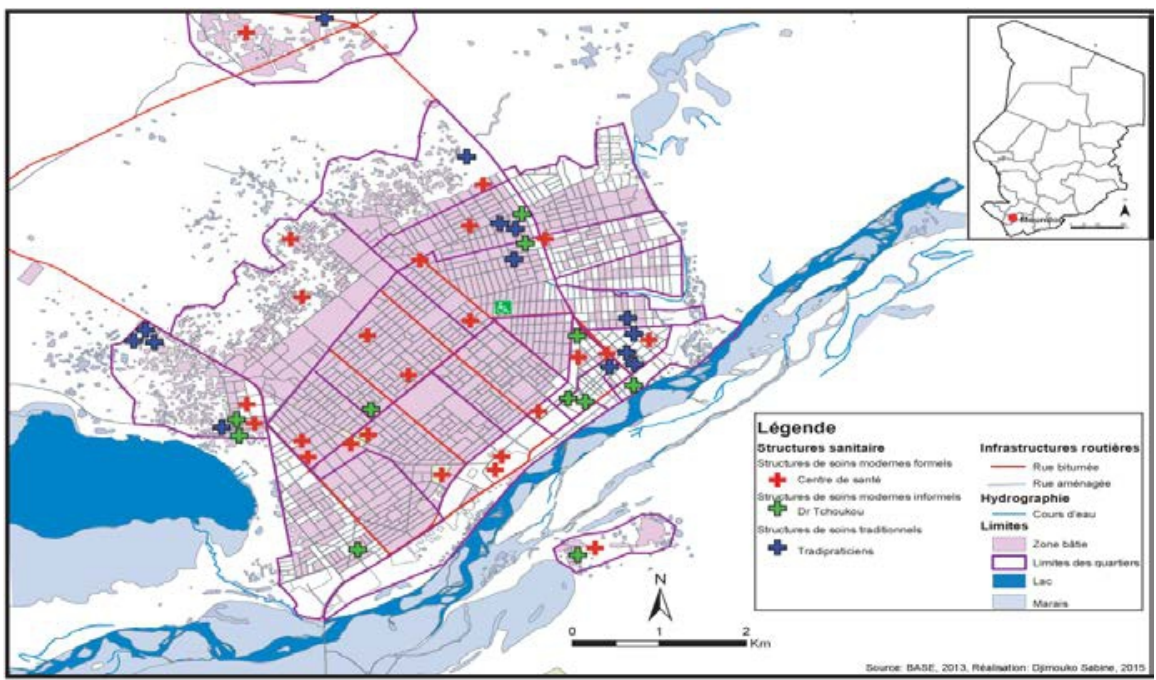

Figure5. Spatialisation de la diversité de l'offre de soins dans la ville de Moundou, chef-lieu de la région d'étude.

Dans la ville de Moundou, les centres de santé modernes ${ }^{8}$ sont concurrencés par la médecine traditionnelle ${ }^{9}$ et le secteur informel de la médecine moderne ${ }^{10}$.Cette diversité d'offres donne aux patients la possibilité de faire son choix: avant de le faire, ce dernier évalue les opportunités que la fréquentation de tel ou tel centre de santé peut lui apporter. Ces raisons peuvent être purement subjectives, ${ }^{11}$ alors que l'organisation de soins est basée sur des principes purement technocrates, c'est à dire un rapprochement physique des soins pour un meilleur accès. À partir des raisons qui ont guidé les choix des patients qu'il nous sera possible de comprendre mieux les déterminants réels d'utilisation des services de santé dans la région.

\section{Les raisons du choix des établissements de soins : un défi pour la planification sanitaire}

Les établissements les plus fréquentés sont ceux qui offrent des opportunités en termes de temps de parcours, et de facilité dans le déplacement

Parmi les raisons ayant motivé les choix du recours aux soins, la proximité domine, tandis que dans leurs trajets, les patients parcourent de

\footnotetext{
${ }^{8}$ Les centres de santé modernes sont représentés par les croix rouges.

${ }^{9}$ Les prestataires de la médecine traditionnelle sont représentés en croix bleu.

${ }^{10}$ L'offre de soins du secteur informel de la médecine moderne est représentée en croix verte. Il concerne les vendeurs des produits pharmaceutiques prohibés. Ils sont appelé communément «Docteurs Djim» ou «Docteurs Choukou».

${ }^{11}$ Le gaie qui influence le choix du patient peut être le coût de déplacement ou de soins, le temps de déplacement, la qualité de soins, l’accueil, etc.
} 
longues distances laissant derrière eux les établissements les plus proches. Alors s'agirait-il d'une proximité relative ? La figure 6 présente les raisons qui guident les choix des patients dans le recours aux soins de santé.

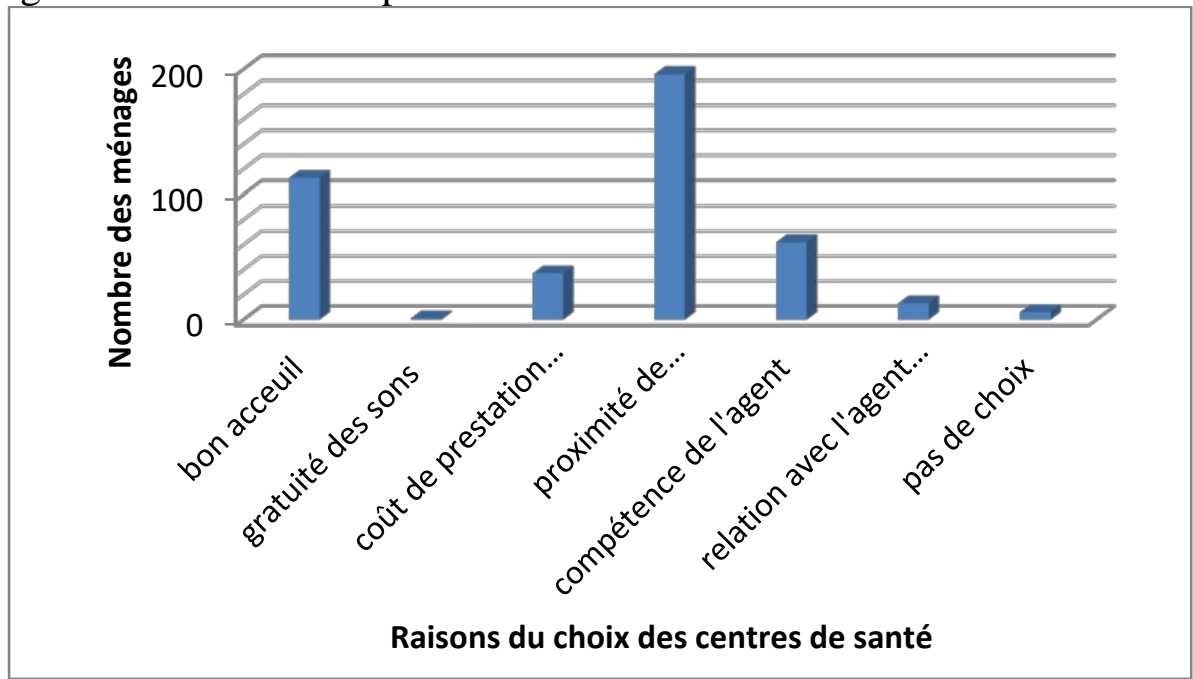

Source : données primaires

Figure 6. Recours aux soins et raisons du choix thérapeutique

En géographie, la distance est relative (Vigneron, 2000). Dans l'analyse des conditions d'accès on parle de plusieurs sortes de distance (financière, sociale, psychologique, et géographique) : ainsi, la distance est ressentie différemment par des catégories sociales ${ }^{12}$. Par ailleurs, la question du recours aux soins et celle de la capacité du patient à utiliser l'offre font intervenir la dimension sociale et rendent complexes les conditions d'accès aux soins telles que présentées dans la figure 6. Le deuxième élément qui motive le recours aux soins est l'accueil qui positionne après la proximité, et la compétence de l'agent. Ces deux éléments (accueil et compétence de l'agent) sont des composantes de la qualité de soins et se rapportent à la subjectivité. Ce qui induit de nouvelles responsabilités de coordination qui incomberont aux districts sanitaires, surtout que cette dimension sociale constitue, une composante essentielle pour l'accès aux soins de santé.

Des travaux scientifiques menés par Michotte P., (1922) et Dahl (1959) cité par Pitte (1993) montrent que le volume des déplacements décroît lorsque le temps de parcours pour atteindre une même destination (lieux de travail, de loisirs, d'implantation d'un service de consommation, etc.)

\footnotetext{
${ }^{12}$ Selon Dumond (2008), la distance peut subir une réduction ou un allongement selon que l'on se déplace à l'aide d'un moyen performant ou non performant. Pour lui la distance ne dépend pas la longueur du trajet mais plutôt la qualité du réseau de communication et du moyen de déplacement.
} 
augmente. Ainsi, toute augmentation de ce temps de parcours pour se rendre à une destination diminuera d'autant son attractivité Dahl (1959) in Pitte (1993). Ces travaux sont largement influencés par la loi des carrés de la force inversement proportionnelle au carré des distances. Ici, il est question d'examiner l'ampleur de la distance sur l'utilisation des services de santé. Newton (1687) $)^{13}$ a affirmé qu'une diminution des mouvements était proportionnelle à l'augmentation de la distance. Dans cette théorie, Newton met en avant le rôle de la distance sur l'ampleur du phénomène observé. La distance est alors jugée suffisante pour mesurer le volume d’échanges ou des flux cependant, Dumont (2008) considère que la distance est un facteur peu contraignant dans les flux. Dans la région d'étude, on réalise que les patients ne sont pas attirés par les centres de santé les plus proches (figure7).

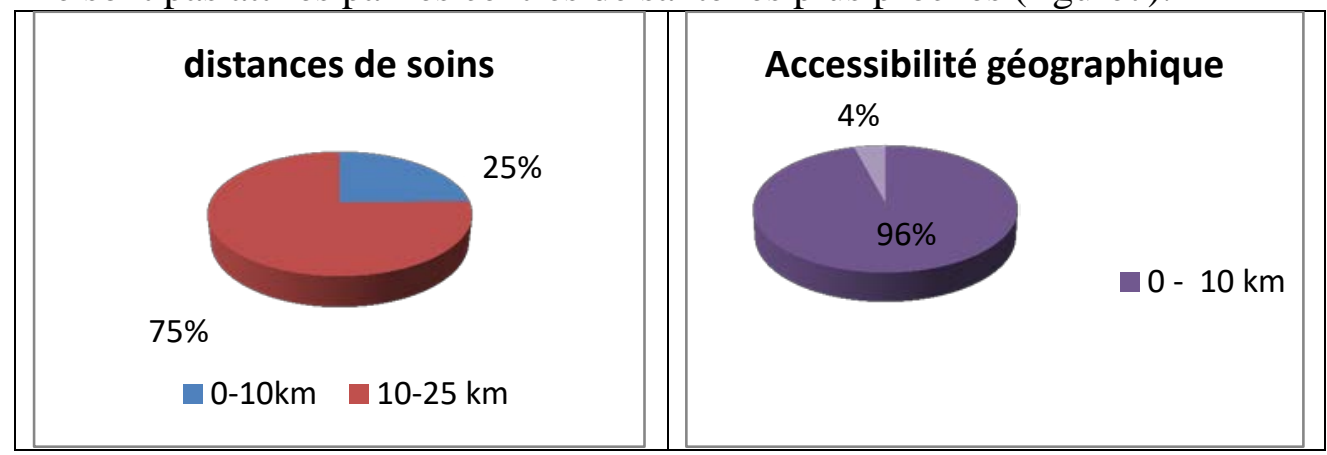

Source : données primaires (ménage, 2013)

Figure7. Indicateur d'accès géographique et distance réelle d'accès aux soins

La planche ci-haut nous présente deux figures côte à côte. Sur la figure de la droite, nous pouvons lire la proportion des individus habitant dans un intervalle allant de 0 à $10 \mathrm{~km}$ d'un centre de santé et ceux qui sont séparés par une distance supérieure à $10 \mathrm{~km}$. On constate que seulement 4\% vit à une distance supérieur à $10 \mathrm{~km}$ d'un centre de santé. À gauche, une figure présentant la proportion des personnes ayant effectuées une distance supérieure à 10 km (75\%) pour se soigner laissant après eux des centres de santé les plus proches vides (figure 7). Ainsi, dans la région du Logone occidental, les établissements les plus proches ne sont pas toujours fréquentés par les patients, ceux-ci préfèrent parcourir les distances de plus en plus grandes à la recherche de la satisfaction. En effet, dans ce cas, la proximité se mesure moins en termes de distance physique que par les possibilités de contact, d'information ou de familiarité avec les lieux. Il arrive que les établissements les plus éloignés physiquement soient les plus accessibles, soit parce qu'ils sont desservis par un bon réseau de circulation, soit parce qu'ils sont joignables par des moyens de déplacement efficace et moins couteux. Ces éléments font que distants, les établissements qui offrent

\footnotetext{
${ }^{13}$ https://fr.wikipédia.org/wiki/Isaac_Newton \#La_loi_universelle_de_la_gravitation
} 
plus d'opportunités d'accès à leur service sont plus sollicités que ceux qui sont à proximité des patients mais dont les conditions d'accès (défectuosité et ou sinuosité des routes, manque de moyens de transport, enclavement) s'érigent en barrière. Selon Vigneron (2000), la distance est relative, car des endroits qui paraissent lointains peuvent être plus accessibles que ceux qui se trouvent à proximité des consommateurs ; c'est l'exemple du trajet entre le centre de Délia situé à $30 \mathrm{~km}$ de Moundou et celui de Mbakoul à $25 \mathrm{~km}$. Pour se rendre au centre de santé de Délia a situé sur la route nationale, on a besoin d'au plus $30 \mathrm{mn}$ pour une vitesse moyenne de $80 \mathrm{~km} / \mathrm{h}^{14}$. Le trajet Mbakoul-Moundou se fait en trois heures d'horloge. Sur cette route, la vitesse est limitée à $8,33 \mathrm{~km} / \mathrm{h}$ à cause de la défectuosité des routes. En effet, pour se rendre à Délia, on peut rouler 10 fois plus vite que quand on se rend à Mbakoul. En dehors des autres raisons subjectives liées à la qualité et à l'adaptabilité des soins, les positions de certains centres de santé peuvent favoriser ou défavoriser leur sollicitation à cause des difficultés liées à leurs conditions d'accès ; celles-ci expliquent pourquoi certains patients parcourent de longues distances au lieu de fréquenter un établissement qui se trouve à proximité, mais dont les conditions de déplacement ne sont pas faciles. Dans l'appréciation de la distance, le temps du trajet est un élément non négligeable dans le recours aux soins. Observons sur cette figure les différentes distances parcourues par les patients de la région en 2013 à la recherche des soins.

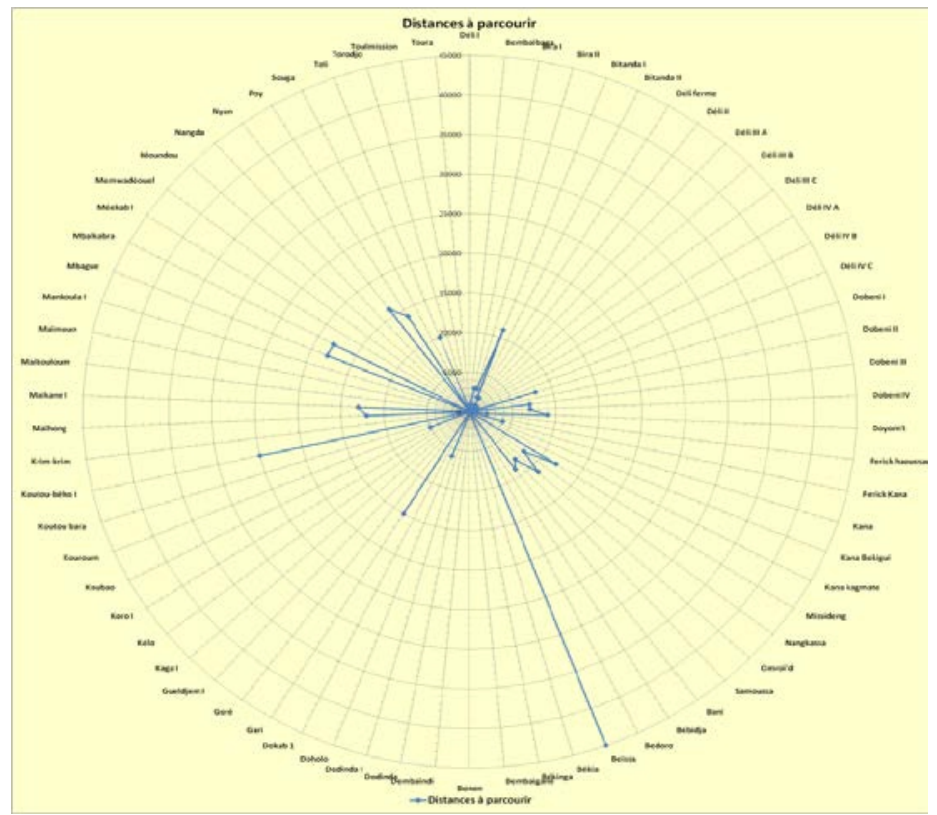

Source : données primaires (enquête ménage 2013)

Figure8. Recours aux soins et distance d'accès

14 Le trajet est effectué en voiture. 
Ce comportement conduit à examiner la relation entre la distance d'accès et la fréquentation des établissements. En effet, la distance n’est pas significativement associée au choix de l'établissement, donc à son utilisation. Les établissements les plus fréquentés sont ceux qui exercent une attractivité par leur compétence mais non par leur proximité.

Les résultats de l'étude révèlent que dans la région d’étude, la distance linéaire, c'est-à-dire la mesure d'écart entre le patient et le centre de santé, n'est pas significativement associée à la fréquentation des centres de santé. Par conséquent, la fréquentation d'un centre de santé est possible si le patient réunit l'ensemble des conditions qui lui permettent de se déplacer même sur une grande distance et d'obtenir les soins dans le temps et dans l'espace. Les centres de santé sont des institutions assurant les services sanitaires destinés à une collectivité donnée. Leur rôle, c'est la prévention des maladies et la promotion de la santé individuelle et collective (OMS, 1999). La construction d'un centre de santé nécessite de gros moyens, par ailleurs, son implantation doit faire l'objet des études pertinentes prenant en compte les paramètres d'accessibilité, afin que son utilisation soit effective. Les services de santé ont la prérogative d'assurer la veille sanitaire, la prévention, les soins curatifs, et les soins de réhabilitation. Ces éléments n’ont pas été pris en compte avant les années 1960 dans la recherche des solutions aux problèmes de santé. À l'époque, l'amélioration de la qualité de santé, la prévention n’étaient pas la vocation première de la géographie médicale développée par Sorre (Le Bras et al., 2004). La géographie de la santé a vu le jour dans les années 1970 sous les auspices de Brunet pour corriger ce qui jusqu’à là est resté comme un manque dans les approches géographiques des questions de la santé (Le Bras et al.,Op. Cit.).

L’utilisation des services de santé devient ainsi une question de moyen de transport, de coût de transport, de la qualité de soins, de la motivation et de la capacité du patient à surmonter les barrières physiques, logistiques et financières. L'ensemble de ces conditions doivent nous amener à prendre en considération les variables sociales (Tollonnier, 1992); par ailleurs, la distance prend une étiquette sociale car chaque catégorie sociale la ressent de différentes manières selon son niveau de vie (Vigneron, 2000). Les patients plus nantis peuvent disposer des moyens de transport plus performants et adaptés, ce qui leur offre la possibilité de parcourir de plus grandes distances à la recherche des soins de leur goût (Dumond 2008). Pour ces derniers, la distance n'est pas un élément contraignant à l'utilisation des centres de santés ; cependant, les démunis sont limités et contraints de se rabattre sur l'offre de proximité. Cette réalité est à l'origine de l'élargissement des zones d'influence de certains centres de santé comme on l’observe sur la figure 9. 


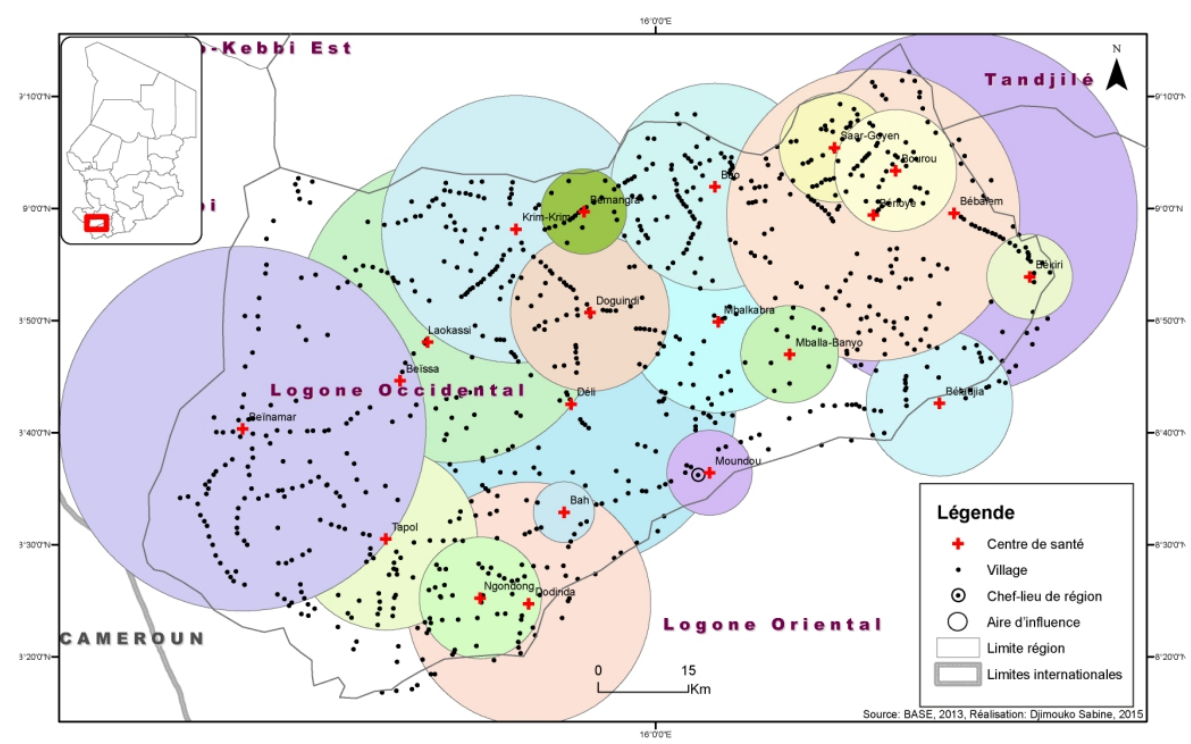

Figure9. Attractivité de certains centres de santé

On observe sur la figure ci-dessus que certains centres de santé ont un espace médical plus élargi que les autres. À cause de leur performance et ou des facilités de déplacement qu'ils offrent aux patients, ils sont capables d'attirer les patients d'autres espaces appartenant aux centres de santé lointain ou voisins. Ainsi, les établissements les plus attractifs sont ceux qui exercent une forte influence ou attraction dans les zones de « recrutement » des autres centres de santé. Pour ces centres de santé, la distance ne peut pas jouer sur leur sollicitation. Il apparait ici évident que la distance comme mesure d'écart entre deux lieux peut être atténuée par la motivation du patient à la recherche d'un soin de qualité et plus adapté.

\section{Les établissements les plus fréquentés sont ceux qui offrent les soins de qualité même s'ils sont joignables à un prix fort}

Pour $78 \%$ des ménages enquêtés, la qualité de soins offerts par les centres de santé est médiocre. Seulement 22\% ont avoué avoir bénéficié de la qualité dans leurs soins (figure9). La qualité de soins est citée comme deuxième raison du choix des établissements de soins. Il est, par conséquent, un élément déterminant pour la fréquentation et l'utilisation des centres de santé. Pour bénéficier de soins de qualité, il faut parcourir de longues distances, cela nécessite de moyens de déplacements adaptés dans un contexte où $68 \%$ des routes sont peu ou difficilement praticables. L'accès aux soins de bonne qualité nécessite des moyens pouvant assurer une certaine capacité (physique, matérielle, financière et psychologique) permettant de surmonter les obstacles et d'atténuer l'effet de la distance. La majorité (75\%), des patients parcourent de longues distances à la recherche 
de la qualité de soins figure 7, page 12). Ceux qui choisissent les établissements éloignés pour se soigner utilisent des engins à moteur tels que les moto-bécanes et voitures (figure 11). Les piétons sont les plus rares à parcourir de longues distances donc disposent de peu de chance d'avoir un soin de bonne qualité. Ainsi, les moyens de déplacement et leur adaptabilité sont associés significativement à l'accès aux soins. Par ailleurs, le moyen qui concourt efficacement à l'utilisation des centres de santé dans la région du Logone occidental sont les engins à quatre roues.

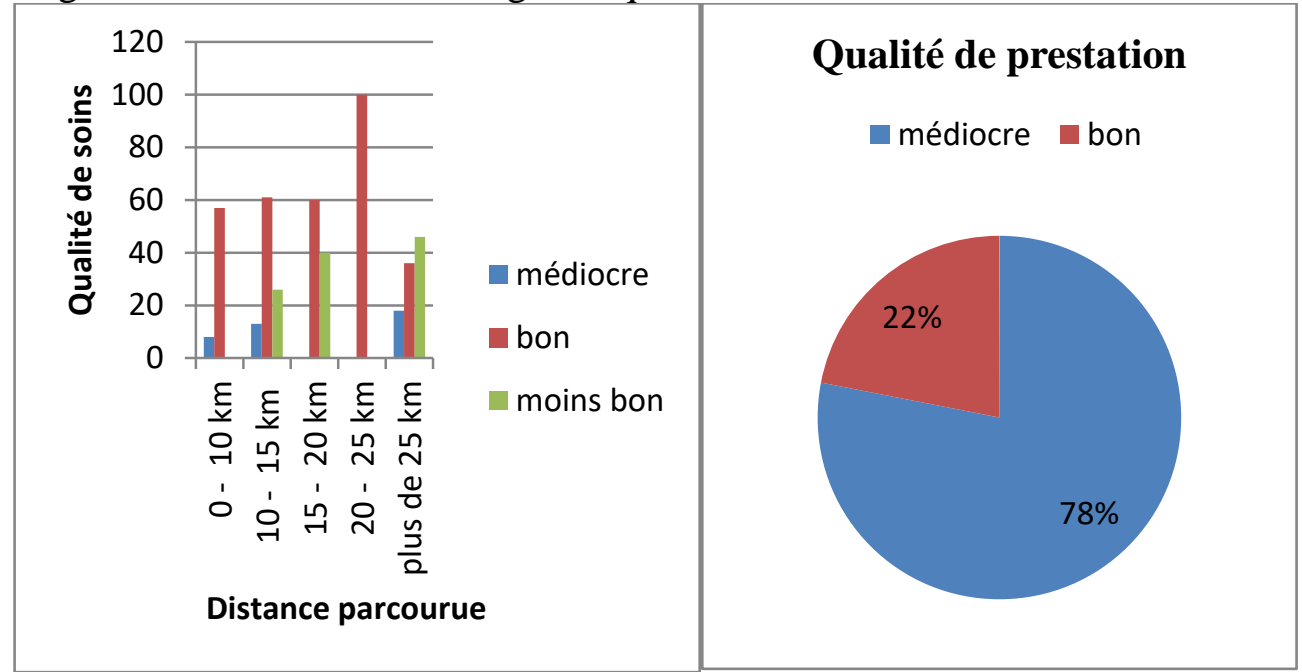

Source : Données primaires (enquête sur les ménages, 2013)

Figure10. Qualité de service et distance d'accès

Il ressort que le moyen de déplacement est un outil qui permet au patient de franchir la barrière de la distance et d'atteindre un établissement quelle que soit la distance. Le fait de posséder un moyen de déplacement peut donner la chance d'accéder aux soins et d'utiliser un service de soins. Par ailleurs, avoir un moyen de déplacement adapté offre encore plus d'opportunité d'accès aux soins de qualité dans la région.

En effet, parmi les différents moyens de transports utilisés, la charrette est le plus utilisé pour le déplacement des patients, toutefois son influence n'est pas significative dans l'utilisation des centres de santé; le fait d'avoir la possibilité de faire usage cet engin n'offre aucune opportunité d'utilisation d'un service de santé en cas de maladie. C'est la voiture qui offre $10 \%$ d'opportunité d'accès aux services de santé à ses usagers. Les autres moyens de déplacement comme la bicyclette, la moto-bécane, le déplacement à pied ou le porte-tous ont également un effet non significatif dans l'utilisation des centres de santé. Les patients qui utilisent les motos sont nombreux à utiliser les services des centres de santé situés à une distance de $20 \mathrm{~km}$. Les longs trajets (plus $25 \mathrm{~km}$ ) sont effectués pour la plupart en voiture. Les utilisateurs de charrettes (figure 11) sont nombreux à 
fréquenter les centres de santé les plus proches ( 0 à $15 \mathrm{~km})$. Ainsi, les piétons et utilisateurs des charrettes qui constituent $78 \%$ des patients ne peuvent parcourir de grandes distances à la recherche de soins. Ainsi les types de moyens de déplacement peuvent limiter le déplacement des patients dans l'espace. En effet, quelles est la relation entre la qualité de soins, les moyens de déplacement et la distance parcourue par les patients?

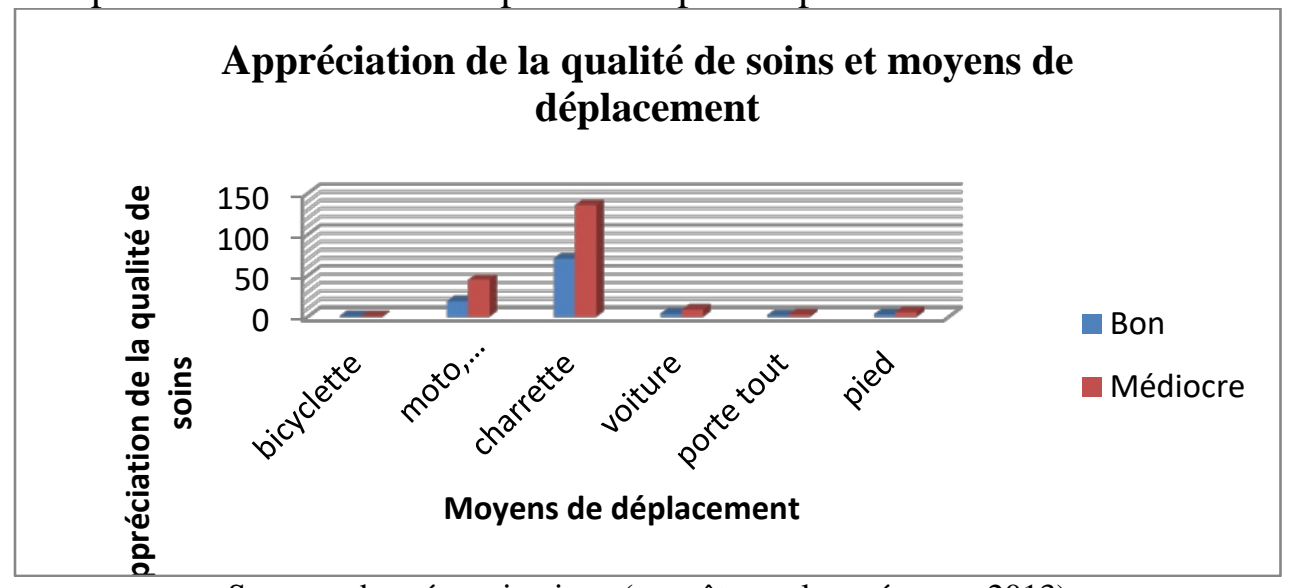

Source : données primaires (enquête sur les ménages, 2013)

Figure11. Appréciation de la qualité de soin et les moyens de déplacement utilisés

Sur la figure 11, les utilisateurs des charrettes sont nombreux à trouver que la qualité de soins est médiocre. Dans la région d'étude, tous ceux qui ont parcouru une grande distance ont obtenu les soins de qualité. Entre la qualité de soins et le moyen de déplacement il existe une relation significative. Par ailleurs, l'analyse de la régression linéaire révèle que parmi les divers moyens de transport utilisés par les patients, la voiture offre plus d'opportunité d'accès que les autres ${ }^{15}$. Pour obtenir un soin de bonne qualité, il faut avoir un moyen de déplacement adapté et être capable de franchir une distance c'est-à-dire, au-delà de $20 \mathrm{~km}$ (figure10, page 14). Les moyens de déplacement, quand ils sont efficaces ne permettent pas seulement d'obtenir la qualité de service de soins, ils offrent d'autres bénéfices comme la réduction du temps et du coût de déplacement des patients qui peuvent être des facteurs limitant l'utilisation des services de santé.

\section{Conclusion}

Le social, le culturel, la géographie et la qualité du service sont les éléments clefs qui déterminent l'utilisation d'un centre de santé. Le rapprochement d'un dispensaire villageois ou son implantation n'empêchera pas les malades de réclamer la guérison au temple des ancêtres ou auprès de

${ }^{15}$ La voiture offre $15 \%$ de chance d'accès à ses utilisateurs 
la statue ou encore " chez docteur Djim » ${ }^{16}$ ni de se déplacer sur de longue distance à la recherche d'un centre de santé qui offre un soin de son goût. La relation entre la distance comme mesure linéaire entre deux points et l'utilisation d'un centre de santé n'est pas significative dans la région du Logone occidental. Les variables qui influencent l'utilisation des centres de santé sont d'ordre sociales (habitudes et comportements dans le choix thérapeutique, niveau de vie, niveau d'instruction), et géographiques (états de la route, de moyens de transport). Ces éléments s’ils sont médiocres s'érigent en véritables barrières et deviennent des facteurs d’allongement du temps de parcours et d'augmentation du coût de déplacement rendant certains centres de santé infréquentables.

Par conséquent, les établissements les plus proches et les produits modernes servis par les centres de santé ne sont pas très sollicités par les patients. Le comportement des patients dans la recherche de soins échappe aux attentes de la gouvernance de la santé: Les stratégies d'accès aux soins développées par la population de la région d'étude nous fait pénétrer dans un autre univers géographique, qui diffère de celui, aseptisé, élaboré administrativement par le planificateur de santé. La fréquentation d'un établissement de soins est basée sur la subjection qui pour la plupart ne répond généralement pas à la logique spatiale largement défendue par les théoriciens occidentaux ${ }^{17}$.

Pour les populations, les établissements les plus accessibles sont ceux qui offrent des soins de leur goût et les plus accessibles en termes de temps de parcours, et de facilité du déplacement. Puisque, la réduction de la distance est loin de tout résorber, il faut remodeler l'organisation de l'offre en introduisant les variables de satisfaction et d'adaptabilité. Ces variables doivent être d'ordre sociologiques (niveau de vie des consommateurs, habitudes et mentalités) et géographiques (climat, nature du sol, saisons, qualité des routes, disponibilité et adaptabilité des moyens déplacements) fournies par nos enquêtes sociologiques et géographiques. Chacune de ces variables doit avoir un poids dans le phénomène étudié (utilisation des structures de santé). Par exemple, la voiture comme moyen de déplacement offre $15 \%$ de chance de plus à un patient d'avoir accès aux soins, le fait de d'avoir un centre de santé dans son milieu de résidence offre également $10 \%$ de chance au patient d'utiliser son service et un établissement qui fournit un soin de qualité contribue à hauteur de $15 \%$ à sa sollicitation. C'est à partir de ces informations qu'il faut établir les priorités en termes d'actions à mener en faveur d'accessibilité et d'une meilleure utilisation des ressources de santé.

${ }^{16}$ Les « docteur Djim » sont des agents non qualifié du secteur informel de la médecine moderne qui font des soins parallèles.

${ }^{17}$ Dahl. (1959)., qui a démontré qu'une diminution des mouvements était proportionnelle à l'augmentation de la distance. 


\section{References:}

1. Barbat-Buissière S., 2008. L'offre de soins en milieu rural: l'exemple d'une recherche appliquée en Auvergne. Thèse de Doctorat Ph.D. Université Blaise-Pascal-Chermont II.423 p.

2. Brunet R.et Jailly J., 2004. Se soigner au Mali : une Contribution des sciences sociales : 12 expériences de terrain. Karthala, Paris, 89 p.

3. Brunet R. etJailly J., 2003. Quel financement pour quels services de santé: Argent et santé dans les pays d'Afrique et d'Asie à faible revenu. Karthala, Paris, pp 97-118.

4. Bugnicourt A, 1990. Disparités régionales et aménagement du territoire en Afrique. Armand Colin, Paris, 335 p.

5. Cabot J., 1965. Le Bassin du Moyen Logone. ORSTOM, Paris, 355 p

6. Djimouko S., 2008. Répartition spatiale des établissements de santé en Afrique subsaharienne. Mémoire de DEA en géographie de la santé, UL, 115 p.

7. Dolfus O., 1976. L'analyse géographique. PUF, Collection Que saisje ? Paris, $142 \mathrm{p}$.

8. DRSLOC., 2012. Rapport annuel, comité directeur. 86 p.

9. DRSLOC., 2013. Rapport annuel, comité directeur. 76 p.

10. Dumond M., 2008. Lire et comprendre les espaces habités. Armand Colin, Paris, 125 p.

11. Doundo R., 1994. Matrice des districts sanitaires du Tchad. Cellule technique des suivis tables Ronde sectorielles "Santé Affaire Sociale » PNUD/OMS/CHD/93/2007, N’Djaména 193 p.

12. INSEED 2013, Enquête Nationale sur les indicateurs du Paludisme au Tchad, Rapport final. INSEED, $80 \mathrm{p}$.

13. Le Bras M, Malvy J-M-D. 2004. Complexe pathogène tropical: Regard nouveau sur un concept ancien. Médecine Tropicale pp. 613-618.

14. Lucas G.V., Tollonnier, E. Vigneron, 1998. Une typologie des paysages socio sanitaires en France. Armand Colin Paris, 95 p.

15. Lucas V., Tonnellier F., 1995. «Évolution départementale de l'offre et de soins médicaux: indicateurs économiques et indicateurs de santé », Espace, populations, sociétés, 1995, nº1, p. 67- 73.

16. Michotte P., 1922. "L'orientation nouvelle en géographie", Bulletin de la Société royale de géographie, 1, p 1-39.

17. Ministère de la Santé Publique 2015. Engagement politique du Chef de l'État de la République du Tchad dans la mise en æuvre de la Politique Nationale de Santé (un regard rétrospectif de 2009 à 2015). N’Djaména, $21 \mathrm{p}$.

18. Ministère de la Santé Publique 2010.ASSTTome A, Tchad, 221 p. 
19. Ministère de la Santé Publique 2006.ASSTTome A, Tchad, 227 p. 20. Ministère de la Santé Publique 2011.ASST Tome A, Tchad, 235 p. 21. Ministère de la Santé Publique 2012.ASST Tome A, Tchad, 265 p.

22. Ministère de la Santé Publique 2013.ASST Tome A, Tchad, 246 p.

23. Nadjihoroum Ndigadet A., 2007. Pauvreté et accès aux soins de santé obstétricaux au Tchad. Mémoire de DESS, IFORD, Yaoundé, 138 p.

24. OMS., 1999. Réduire la mortalité maternelle, une déclaration commune OMS/ UNFPA/UNICEF/Banque Mondiale. OMS, Genève $45 \mathrm{p}$.

25. OMS., 1991. L'engagement communautaire en faveur du développement sanitaire : un défi pour les services de santé. Genève, $448 \mathrm{p}$.

26. Picheral H., 1976. Espace et santé, géographie médicale du Midi de la France. $425 \mathrm{p}$.

27. Picheral H., 1982. Géographie des maladies, Géographie médicale, géographie de la santé. L'Espace Géographique, n³, pp.161-175.

28. Picheral H., 1984. Médecine libérale et espace médical en France : spécialisations et besoins. In : Médicomérie régionale, Anthropos, Paris, pp. 33-57.

29. Picheral H., 1985. Mots et concepts de la géographie de la santé. Cahier Géo, n², 45 p.

30. Picheral H., 1989. La desserte en soins médicaux : Variations sociogéographiques. Cahiers GEOS, $\mathrm{n}^{\circ} 16$, Actes du colloque, Montpellier, $82 \mathrm{p}$.

31. Picheral H., 1997. La valeur stratégique de l'espace dans la politique sanitaire. Dossier santé publique, $\mathrm{n}^{\circ} 19$,pp. 35- 36.

32. Picheral H., 1998. Territoire et valeur d'usage de l'espace : éléments d'une géographie des soins. Actes du 5e colloque de géographie et sociologie économique de la santé. CREDES, Paris, pp. 39-45.

33. Pitte J-R., 1993. Géographie, Classe de seconde. Nathan, France, 288 p.

34. Salem G., 2013. Géographie et sciences de la santé. Université de Paris Ouest, Paris, 80 p.

35. Salem G., 1998. La santé dans la ville, Géographie d'un petit espace dense, Pikine (Sénégal). Karthala- ORSTOM, Paris, 360 p.

36. Salem G., 1999. Les problèmes de santé dans les villes : une urgence négligée. Médecine tropicale, Marseille, vol.53, no 2, pp. 31-33.

37. Tollonnier F., 1992. Inégalité géographiques et santé : Évolution depuis le XIX $^{\text {ème }}$ siècle en France. Biblio nº 922, CREDES, Paris, 84 P. 
38. Vidal de la Blache P., 1902. Les conditions géographiques des faits sociaux, in Annales de géographie, vol. XI, pp.13-23.

39. Vigneron E. et Brau F., 1960. Approches géographiques de la planification sanitaire. GEOS, Montpellier, pp. 46-72.

40. Vigneron E., 2000.Santé publique et aménagement du territoire, Actes FIG 2000.

41. Vigneron E., 2000. Territoire et santé. Documentation française. Paris cedex, 64 pages.

42. Vigneron E., 2000. Santé publique et aménagement du territoire. Actes du 11e Forum International de Géographie, 213 p.

43. https://fr.wikipédia.org/wiki/Isaac_Newton \#La_loi_universelle_de_l a_gravitation. 